\title{
Interval Type-2 Beta Fuzzy Near Sets Approach to Content Based Image Retrieval
}

\author{
Yosr Ghozzi, Student Member, IEEE, Nesrine Baklouti, Senior Member, IEEE, Hani Hagras, Fellow, IEEE, \\ Mounir Ben Ayed and Adel M. Alimi, Senior Member, IEEE
}

\begin{abstract}
In computer-based search systems, similarity plays a key role in replicating the human search process which underlies many natural abilities, such as image recovery, language comprehension, decision making, or pattern recognition. The search for images consists in establishing a correspondence between the available images and those sought by the user, by measuring the similarity between the images. In fact, image search per content is generally based on the similarity between the visual characteristics of the images. The distance function used to evaluate the similarity between images depends notonly on the criteria of the search but also on the representation of the characteristics of the image. This is the main idea of a contentbased image retrieval (CBIR) system. In this article, we first constructed type-2 beta fuzzy membership of descriptor vectors to help manage inaccuracy and uncertainty of the characteristics extracted from the feature of images. Subsequently, the retrieved images are ranked according to the novel similarity measure, which is noted type-2 fuzzy nearness measure (IT2FNM). By analogy to Type-2 Fuzzy Logic, and motivated by a near sets theory, we advanced a new fuzzy similarity measure (FSM) noted as interval type-2 fuzzy nearness measure (IT-2 FNM). Then, we proposed three new IT-2 FSMs and provided mathematical justification to demonstrate that the proposed FSMs satisfy proximity properties (i.e. reflexivity, transitivity, symmetry, and overlapping). The experimental results generated using three image databases show consistent and significant results.
\end{abstract}

Index Terms - Interval-Type-2 Fuzzy Sets, Near Sets, Function Beta, Fuzzy similarity measure, CBIR.

\section{INTRODUCTION}

C ONTENT-based image retrieval (CBIR) is one of the basic research challenges that have been studied in depth by the multimedia community for decades, due to its wide range of applications in the search for information and "computer vision", "Database Management", "ManMachine interface" [1]. Over the years, CBIR systems have been used to efficiently retrieve relevant images from a large set of databases. CBIR techniques recover images visually similar to a given query image. Therefore, several

Y. Ghozzi, N. Baklouti, M. Ben Ayed and A. M. Alimi are with the Research Groups in Intelligent Machines (REGIM-Lab), University of Sfax, National Engineering School of Sfax (ENIS), BP 1173, Sfax, 3038, Tunisia (e-mail: $\quad$ yosr.ghozzi@ieee.org; $\quad$ nesrine.baklouti@ieee.org; mounir.benayed@ieee.org; adel.alimi@ieee.org).

H. Hagras is Director of the Computational Intelligence Centre and the Head of the Fuzzy Systems Research Group in the University of Essex, UK (e-mail:hani@essex.ac.uk).
CBIR systems have been developed for image retrieval that represent promising solutions, such as the QBIC image and video content system [2], VisualSEEk [3] and SIMPLIcity [4], etc. Today, CBIR systems continue to face the challenge of the semantic divide in the accuracy of the relevant image and the subjectivity of human perception of visual content due to the incorrect selection of feature extraction methods and the measure of similarity. The effectiveness of such CBIR systems, therefore, depends, among other things, on a better match between the machine and humans, in the mode of representation and description of the information contained in the images. These methods are intended to reduce the notion of visual similarity between images to a simple notion of proximity between descriptors. Besides, the degree of similarity between the images is measured according to the characteristic descriptors which describe the visual content of an image. These features are coded to improve recovery performance using vector descriptors. On the other hand, a review of the most difficult problems of the CBIR has been proposed in [1, [5- -9 . Although the number of CBIR methods is very large, the functionalities that are designed by the man based on these engineering skills and domain expertise, are difficult to be accurately described by the machine. The search for images similar to a request image is equivalent to searching for neighbors closest to the descriptor of the request image in the description space. In this context, Peters in [10] presented an approach using near sets and tolerance classes. This method is developed within the framework of perceptual systems, where each image or part of an image is considered as a perceptual object 11]. The central idea of the near sets is the research of the similarity between sets of disjoint objects. Thus, the recovery task can be considered as the similarity between sets of objects based on their descriptions. A tolerance measure of nearness (similarity) TNM was introduced in 12, which was applied to problems of image analysis and CBIR [13]-17]. It was proven that TNM may perform as better than other measures in many real-world applications. In recent years, there have been countless similarity studies in different image processing applications. A summary of several measures is detailed in [18]-23. One of the solutions allowing to approach more faithfully the human way of thinking, which is generally characterized by imprecision and ambiguity, is the use of fuzzy logic, which makes it possible to model these characteristics and reduce ambiguity. Fuzzy logic allows 
a gradual transition between the equality of descriptions. Therefore, the integration of near sets with fuzzy sets provides a consistent balanced mix for the development of an efficient soft computing strategy in computer vision. The motivation behind the fuzzy near sets is to define the fuzzy similarity between sets of objects based on their fuzzy description. In fact, according to Peters in 24] a Fuzzy Near set was proposed to represent the similarity between two images. The practical outcomes of the used fuzzy near sets approach in our work [14], show the improvements that can be achieved compared to the results obtained by near sets, but still insufficient compared to the current work. Due to the complexity of the image visual structures, it was found that ambiguity might not be taken with type1 fuzzy sets, so, the use of type- 2 fuzzy logic would be necessary to manage the uncertainty that exists in realworld problems 25]. As type-2 fuzzy sets provide us with more design degrees of freedom, according to Mendel. These Type-2 fuzzy sets are very useful when it is difficult to determine an exact membership function as in Type1 fuzzy sets 26]. However, when there is no membership uncertainty, the set is automatically reduced to a Type- 1 fuzzy set while Type-2 fuzzy sets are now well established and are gaining more and more popularity. Therefore, the use of type- 2 fuzzy near sets seems suitable for the paper context. Actually, the image retrieval by using Interval Type-2 Fuzzy Logic has been proven to be a great success in a large variety of applications, such as [27]-[29], among the works can find some: Xing and al. in [30] have been proposed an interval type- 2 fuzzy clustering method based on neighborhood information to improve the classification accuracy of remote sensing images with complex land cover. For cancer diagnosis and prognosis, Singh et al. proposed in 31] a robust feature extraction approach based on the principal component analysis with interval type-2 fuzzy membership functions (IT-2PCA). Moreover, in 32], researchers proposed a novel way using the concept of footprint of uncertainty in interval type- 2 fuzzy sets. This method can handle the veracity characteristic issue of the big data and reduce the instances to a manageable extent. With the edge-detection method, [33], [34], Patricia used the morphological gradient technique and generalized type-2 fuzzy logic, while Gonzalez applied the interval type-2 fuzzy sets on fuzzy images. In fact, previous literature suggested that interval type-2 fuzzy sets (IT2FS) can offer an alternative that can handle vagueness and uncertainty. The paper of Mittal et al. 35 offers a comprehensive review of the most relevant work in the framework of type-2 fuzzy logic, with its theoretical and practical implications. Castillo in 25] presented a literature review of recent applications using type- 2 fuzzy systems based on image processing. One of the most interesting topics in the FS theory is the definition of the measure of similarity between FSs, to indicate their degree of closeness. In this context, several type 2 fuzzy similarity measures (T-2 FSMs) were introduced where $\mathrm{Wu}$ and Mendel in 36. presented a comprehensive overview of existing similarity measures for general type-2 FSs and proposed the Jaccard similarity measure for these sets. A comparative study between type 1 and type 2 FSM can be found in 37. Three new Interval Type-2 Fuzzy Similarity measures (IT2 FSMs) between Intuitionistic Fuzzy Sets were exposed by Cherif et al in [38]. In this paper, we propose a new Interval Type-2 Beta Fuzzy Near sets approach to Content based Image Retrieval. We first proceed with the feature vectors extraction of all images in the database. Then, the fuzzification of descriptor vectors applied by using the interval type- 2 beta fuzzy membership function which deals with the uncertainty of feature extraction characteristics from images. Near sets theory, aims at grouping the images according to some common features, we compare the feature vector of the query image to another image to find the nearest objects. By analogy to Type-2 Fuzzy Logic and being motivated by Henry' study in [16], we advanced then three new interval type-2 fuzzy similarity measures. Since a relevant similarity measure has to fulfill the properties of reflexivity, transitivity, symmetry and overlapping, mathematical demonstrations were provided for our proposed similarity measures. Experimental results on four image databases; SIMPLIcity, Corel-1k, Caltech101, and Image-Net have been ensured. Within this approach our main contributions are summarized as follows: 1) The development of a new description vector for the images by using an interval type- 2 beta TFS (IT2 BFS), which enable gradual transition descriptions.

2) The new use of the near-fuzzy set theory that tolerates the equality of descriptions when comparing elements based on their descriptions.

3) Three new similarity measures between IT-2 fuzzy sets are advanced to obtain a superior approximation and similarity estimation among images.

The rest of this paper is organized as follows: Section II presents a brief overview of the previous work employing type-2 fuzzy sets and near sets to the fields of imagebased image retrieval. Section II displays the theoretical foundations of fuzzy sets and near sets. While section four, is devoted to the presentation of the sought objectives pursued and to the adopted research methodology. The experiments and results are presented in section five. Finally, a conclusion is presented.

\section{PRELIMINARIES}

This section presents the basic theoretical concept behind the methodologies used in this research.

\section{A. Near Sets basis}

Near sets gather disjoint sets each other [39]. Whenever there are observable similarities between the objects in the sets. The similarity is determined by comparing lists of object feature values which represent an object. A probe function is a real assessed function that represents these characteristics (features). Tolerance relationship gives an intransitive idea about the world [12]. Tolerance near sets is identified by a tolerance relationship based on descriptions. A perceptional system is a group of perceived 
items associated with a group of probe functions. And a perceptual item in a conceptual system can be described as follows. Let $O$ represent a set of perceptual objects, and let $B$ denote a set of real-valued functions, denoted probe functions, representing object features, and let $\phi(x) \in B$, where $\phi_{i}(x): \underline{O} \longrightarrow \Re$. In combination, the functions representing object features provide a vector containing measurements (returned values) for an object description, associated with each functional value $\phi_{i}(x)$ for $x \in X$, where $|\phi|=l$; i.e. the description length is $l$.

\section{Object Description:}

$\phi_{B}(x)=\left(\phi_{1}(x), \phi_{2}(x), \ldots, \phi_{i}(x), \phi_{1}(x)\right)$.

As fellows, the relationship between objects is determined by the probe functions in $B$. Our senses are defined to probe functions. In the tolerance space, a specific tolerance relation defined in 12 is given by:

\section{Definition:}

Let $\langle O, F\rangle$ be a perceptual system and let $\epsilon \in \Re_{0}^{+}$(real). For every $B \subseteq F$ the weak tolerance relation $\cong_{B, \epsilon}$ is defined as:

$$
\cong_{B, \epsilon}=\left\{(x, y) \in O \times O\left|\exists \phi_{i} \in B \cdot\right| \phi_{i}(x)-\phi_{i}(y) \|_{2} \leq \epsilon\right\}
$$

where $\|.\|_{2}$ is $L^{2}$ norm.

Notice, the relation $\cong_{B, \epsilon}$ is symmetric and reflexive but not transitive. This relation is very important in finding near sets, since it characterizes tolerance classes within a threshold $\epsilon$.

Finally, the concept of near sets is established on the propositions requiring neighbourhoods and tolerance classes. The separated classes that incorporate similar items are said to be neighbors. The idea behind the nearness measure of Henry and Peters has sought the level of similarity between two variables by omitting the existing correlation between the set of variables, called the tolerance classes. The similarity measures can be grouped into equivalence classes of measures.

Definition : The tolerance nearness measure

Let $\langle O, F\rangle$ be a perceptual system, with $\epsilon \in \Re_{0}^{+}$and $B \subset F$. Moreover, let $X, Y$ be two disjoint sets and $Z=X \cup Y$. A tolerance nearness measure between two sets $X$ and $Y$ is determined by:

$$
\begin{aligned}
& t N M \cong_{B, \epsilon}(X, Y)=1-(\underbrace{\sum_{C \cap}}_{C \in H_{B, \epsilon}(Z)}|C|)^{-1} . \\
& \underbrace{}_{C \in H_{B, \epsilon}(Z)}|C| \frac{\min (|C \cap X|,|C \cap Y|)}{\max (|C \cap X|,|C \cap Y|)})
\end{aligned}
$$

where $H \cong_{B, \epsilon}(Z)$ is the set of tolerance classes.

\section{B. Interval Type-2 Beta Fuzzy basis}

In the previous section, it was shown how tolerance relation can be used in modeling the existing imprecision in human visual perception as well as the fuzzy approach. The most important part of the fuzzy logic theory is the modification of the membership values using various fuzzy techniques. The main importance of the Beta functions lies essentially in its capacity to approximate many usual functions (triangular, trapezoidal, or gaussian shapes) [40]. The concept of a type-2 fuzzy sets (T2 FSs) was an extension of the concept of an T1 FS. T2 FSs are now well established and are gaining more and more in popularity. In this work, the type-2 beta function is chosen for modeling of the fuzzy sets.

Definition: A type-2 fuzzy set, denoted $\tilde{A}$, is characterized by a type- 2 membership function $\mu_{\tilde{A}}(x, u)$, where $x \in X$ and $u \in J_{x} \subset[0,1]$.

$$
\tilde{A}=\int_{x \in X} \int_{u \in J_{x}} \mu_{\tilde{A}}(x, u) /(x, u) J_{x} \subset[0,1]
$$

where $0 \leq \mu_{\tilde{A}}(x, u) \leq 1$ and $J_{x}$ is the closure of $\mu_{\tilde{A}}(x, u)>0$. For any given $x \in X$,

$$
\mu_{\tilde{A}}(x)=\int_{u \in J_{x}} \mu_{\tilde{A}}(x, u) / u
$$

This is a second membership function, that is clearly a type-1 fuzzy set. The Uncertainty in the primary MF consists of the union of all MFs. This Uncertainty represents a bounded region that we call the Footprint of Uncertainty (FOU). This region represents a complete description of an IT2 FS. An IT2 is delimited by two Mfs noted the Upper MF (UMF), which is denoted $\bar{\mu}_{\bar{A}}(x)$ and the Lower MF (LMF), which is denoted $\mu_{\underline{A}}(x)$, i.e.,

$$
\begin{gathered}
F O U(\tilde{A})=\bigcup_{x \in X} u \in J_{x} ; J_{x}=\left[\bar{\mu}_{\bar{A}}(x), \underline{\mu}_{\bar{A}}(x)\right] ; \forall x \in X \\
\bar{\mu}_{\bar{A}}(x) \equiv \bar{F} O U(\tilde{A}) ; \underline{\mu}_{\bar{A}}(x) \equiv \underline{F} O U(\tilde{A}) ; \forall x \in X
\end{gathered}
$$

As the Beta primary MF has four variables, we used the Interval type-2 Beta MF with uncertain center $c \in\left[c_{1}, c_{2}\right]$, a fixed width $\sigma, p$ and $q$, and as is expressed in [41]:

$$
\beta(x)=\left(1+\frac{(p+q)(x-c)}{\sigma p}\right)^{p}\left(1-\frac{(p+q)(c-x)}{\sigma q}\right)^{q}
$$

However, the upper and lower membership functions can be expressed by respectively:

$$
\begin{gathered}
\bar{\mu}_{\tilde{\beta}}(x)= \begin{cases}\beta\left(x ; c_{1}, \sigma, p, q\right) & x<c_{1} \\
1 & c_{1}<x<c_{2} \\
\beta\left(x ; c_{2}, \sigma, p, q\right) & x>c_{2}\end{cases} \\
\bar{\mu}_{\tilde{\beta}}(x)= \begin{cases}\beta\left(x ; c_{1}, \sigma, p, q\right) & x \leq\left(c_{1}+c_{2}\right) / 2 \\
\beta\left(x ; c_{2}, \sigma, p, q\right) & x>\left(c_{1}+c_{2}\right) / 2\end{cases}
\end{gathered}
$$

\section{Fuzzy Near Sets}

As was mentioned by Peters in 42], [43]: A fuzzy set $\mathrm{X}$ is a near set relative to a set $\mathrm{Y}$ if the grade of membership of the objects in sets $X, Y$ is assigned to each object by the same membership function $\phi$ and there is a least one pair of objects $x, y \in X \times Y$ such that $\|\phi(x)-\phi(y)\|_{2} \leq \epsilon$, i.e., the description of $\mathrm{x}$ is similar to the description $\mathrm{y}$ within some $\epsilon$.

Proposition: Fuzzy sets $(X, \phi),(Y, \phi)$ are near sets if, and only if there exists at least one tolerance class $x / \cong_{\phi, \epsilon}$ 
in $(X, \phi)$ and $y / \cong_{\phi, \epsilon}$ in $(Y, \phi)$ such that $x / \cong_{\phi, \epsilon} \triangleright \triangleleft_{\phi, \epsilon}$ $y / \cong_{\phi, \epsilon}$ [43. This model of near fuzzy sets was used in our paper in [14].

\section{Interval type-2 Fuzzy similarity measure IT2FSM}

A similarity measure between two sets (objects) is usually measured using by quantification of objects' attributes. Various similarity measures e.g. Jaccard defined in [37, S1, $\mathrm{S} 2$, proposed in 38 measures are applied between interval type-2 fuzzy sets to define the similarity score. In this section, we briefly present expressions of these similarity measures between type-2 fuzzy sets.

\section{Jaccard's Type-2 Similarity Measure:}

Let $\tilde{A}$ and $\tilde{B}$ be interval type-2 fuzzy sets (IT-2 FSs). A Jaccard Similarity measure 37 between two IT-2 FSs denoted as IT2FSMj (A, B) is presented as follows:

$$
\begin{array}{r}
F S M_{J}(\tilde{A}, \tilde{B})=\frac{p(\tilde{A} \cap \tilde{B})}{p(\tilde{A} \cup \tilde{B})} \\
=\frac{\int_{x} \min \left(\bar{\mu}_{\tilde{A}}(x), \bar{\mu}_{\tilde{B}}(x)\right) \mathrm{d} x+\int_{x} \min \left(\underline{\mu}_{\tilde{A}}(x), \underline{\mu}_{\tilde{B}}(x)\right) \mathrm{d} x}{\int_{x} \max \left(\underline{\mu}_{\tilde{A}}(x), \underline{\mu}_{\tilde{B}}(x)\right) \mathrm{d} x+\int_{x} \max \left(\underline{\mu}_{\tilde{A}}(x), \underline{\mu}_{\tilde{B}}(x)\right) \mathrm{d} x}
\end{array}
$$

where $p(\tilde{A} \cap \tilde{B})$ and $p(\tilde{A} \cup \tilde{B})$ are the cardinalities of $\tilde{A} \cap$ $\tilde{B}$ and $\tilde{A} \cup \tilde{B}$, respectively. The Jaccard type- 2 similarity measure, satisfies the four similarity properties; reflexivity, symmetry, transitivity and overlapping.

Cherif's Interval Type-2 Fuzzy Similarity Measures:

Cherif et al. in 38 have generated new IT2FSM as an extension of the Baccour's distance proposed in 44 and named as IT2FSM1 and IT2FSM2.

$$
\operatorname{IT}_{2 F S} M_{1}(\tilde{A}, \tilde{B})=1-\frac{(\bar{s}+\underline{s})}{2}
$$

with

$$
\bar{s}=\frac{1}{2 n} \sum_{i=1}^{n}\left(\frac{\left|\bar{\mu}_{\tilde{A}}\left(x_{i}\right)-\bar{\mu}_{\tilde{B}}\left(x_{i}\right)\right|}{\bar{\mu}_{\tilde{A}}\left(x_{i}\right)+\bar{\mu}_{\tilde{B}}\left(x_{i}\right)}+\frac{\left|\bar{\mu}_{\tilde{A}}\left(x_{i}\right)-\bar{\mu}_{\tilde{B}}\left(x_{i}\right)\right|}{2-\bar{\mu}_{\tilde{A}}\left(x_{i}\right)-\bar{\mu}_{\tilde{B}}\left(x_{i}\right)}\right)
$$

and

$$
\underline{s}=\frac{1}{2 n} \sum_{i=1}^{n}\left(\frac{\left|\underline{\mu}_{\tilde{A}}\left(x_{i}\right)-\underline{\mu}_{\tilde{B}}\left(x_{i}\right)\right|}{\underline{\mu}_{\tilde{A}}\left(x_{i}\right)+\underline{\mu}_{\tilde{B}}\left(x_{i}\right)}+\frac{\left|\underline{\mu}_{\tilde{A}}\left(x_{i}\right)-\underline{\mu}_{\tilde{B}}\left(x_{i}\right)\right|}{2-\underline{\mu}_{\tilde{A}}\left(x_{i}\right)-\underline{\mu}_{\tilde{B}}\left(x_{i}\right)}\right)
$$

IT2FSM1 measure satisfies the four analogy properties.

$$
\operatorname{IT2FSM}_{2}(\tilde{A}, \tilde{B})=1-\frac{(\bar{s}+\underline{s})}{2}
$$

with

$$
\bar{s}=\frac{1}{2 n} \sum_{i=1}^{n}\left(\frac{\left|\bar{\mu}_{\tilde{A}}\left(x_{i}\right)-\bar{\mu}_{\tilde{B}}\left(x_{i}\right)\right|}{\bar{\mu}_{\tilde{A}}\left(x_{i}\right)+\bar{\mu}_{\tilde{B}}\left(x_{i}\right)}+\frac{\left|\bar{\mu}_{\tilde{B}}\left(x_{i}\right)-\bar{\mu}_{\tilde{A}}\left(x_{i}\right)\right|}{\bar{\mu}_{\tilde{A}}\left(x_{i}\right)+\bar{\mu}_{\tilde{B}}\left(x_{i}\right)}\right)
$$

and

$$
\underline{s}=\frac{1}{2 n} \sum_{i=1}^{n}\left(\frac{\left|\underline{\mu}_{\tilde{A}}\left(x_{i}\right)-\underline{\mu}_{\tilde{B}}\left(x_{i}\right)\right|}{\underline{\mu}_{\tilde{A}}\left(x_{i}\right)+\underline{\mu}_{\tilde{B}}\left(x_{i}\right)}+\frac{\left|\underline{\mu}_{\tilde{B}}\left(x_{i}\right)-\underline{\mu}_{\tilde{A}}\left(x_{i}\right)\right|}{\underline{\mu}_{\tilde{A}}\left(x_{i}\right)+\underline{\mu}_{\tilde{B}}\left(x_{i}\right)}\right)
$$

IT2FSM2 measure satisfies only reflexivity, transitivity, and overlapping properties.

Subsequently, based on the verified properties, IT2FSM1 and IT2FSM2 are Fuzzy Similarity Measures.

Fuzzy Similarity Measure Properties:

Definition: The properties of a fuzzy similarity measure between three sets $A, B$ and $C$ of $F S(X)$ are proposed in [38], 37], [44], as follows:

- P1. Reflexivity: If $S(A, B)=1$ then $A=B$

- P2. Symmetry: $S(A, B)=S(B, A)$

- P3. Transivity: If $A<B<C$ then $S(A, B)>S(A, C)$ and $S(B, C)<S(A, C)$

- P4. Overlapping: If $A \cap B=\varnothing$ then $S(A, B)>0$ otherwise $S(A, B)=0$

A similarity value can vary from -1 to 1 . For two fuzzy sets $\mathrm{A}$ and $\mathrm{B}$, a unit similarity measure means that the two sets are similar; and when the similarity value is -1 that means that the two sets are exactly opposite.

\section{A PROPOSED INTERVAL-TYPE-2 BETA FUZZY NEAR METHOD (IT2BFNM) IN IMAGE RETRIEVAL SYSTEM}

This section describes the content-based image retrieval system used. The proposed work can be well defined by four main steps. Fig. 1 shows a block diagram. As

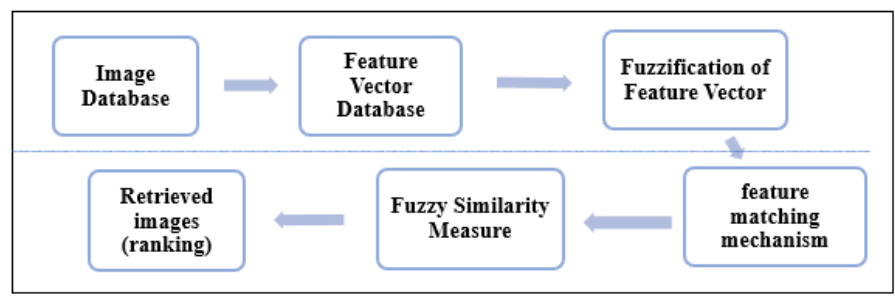

Fig. 1. The strategy of the methodology of work

soon as images are collected and associated and the feature vectors are extracted, the fuzzification process is applied. Then, the resemblance between feature vectors will be computed using the matching mechanism according to the near sets theory. Finally, similarity measures will be calculated to retrieve the nearest images. Those steps are described by the following algorithm:

\section{Proposed algorithm}

1) For each image of database, extract feature vector

2) For each image, apply fuzzification of feature vector

3) For each feature vector, combine the feature vectors of the query and any feature vector of database

4) Apply the feature matching mechanism of the combination of two feature vectors (see [14])

5) Measure the Fuzzy Nearness Similarity

6) Repeat steps 3-5 for each image with all images of database

7) Rank images with Fuzzy similarity Measure 


\section{A. Pre-processing database}

1) Partitioning Image: This research is based on a settheoretic approach to image analysis where each image is viewed as a set of visual elements (describable objects). Each visual element is a part of the image that can be visually perceived and described. However, we decided to decompose the images of our base into blocks of a fixed size where each block is approximately assimilated to a subimage. To control this point, a study has been undertaken in 14.

2) Feature Vector Database: In Near Set theory, an element visual is those that represent something in the physical world, and hence they can be perceived and described. Describing the element is possible through a set of characteristics (features). A visual element is a subimage (as described in the previous section) that can be perceived and described by a probe function (color, shape, or texture). However, in this step, a visual descriptor has to be generated automatically. The choice of the probe function is often detailed in 14 .

3) Fuzzification of feature vector: In this step, each subimage is associated with a fuzzy function that assigns a value (between 0 and 1) to each feature of vector. The building or choosing a proper membership function Interval Type-2 Beta Fuzzy function is described in the previous section.

\section{B. Feature matching mechanism}

The next step consisting to compare the features of each image to any image feature in the database using a tolerance fuzzy relation to obtain a satisfactory matching in near sets sens. Amir in 45] defined a tolerance fuzzy relation $\cong_{\phi, \epsilon}$ between feature vectors. A fuzzy relation $\hat{R}$ is a 'fuzzy set' defined as follows where the membership function represents the degree of membership of each pair of elements in the relation. Furthermore, a tolerance fuzzy relation is a relation that is reflexive, symmetric, and transitive.

Definition: Let $\mathrm{O}$ a set of describable objects, $\mathrm{B}$ a set of probe functions and $\Phi_{B}$ is the set of feature vectors. Suppose $\|\cdot\|_{2}$ is a distance function on $\left(\Phi_{B}, d\right)$. Let $\epsilon<\epsilon^{\prime} \in \Re$. A perceptual fuzzy tolerance relationship $\hat{\cong}_{B, \epsilon}: O \times O \longrightarrow[0,1]$ is defined as follows:

$$
\begin{aligned}
& \hat{\cong}_{B, \epsilon} \\
& =1 \\
& =\frac{\epsilon \prime-\|\left(\Phi_{B}(x), \Phi_{B}(y) \|_{2}\right.}{\epsilon I-\epsilon} \\
& =0 \\
& \text { if } \|\left(\Phi_{B}(x), \Phi_{B}(y) \|_{2}<\epsilon\right. \\
& \text { if } \epsilon<\|\left(\Phi_{B}(x), \Phi_{B}(y) \|_{2}<\epsilon \prime\right. \\
& \text { otherwise }
\end{aligned}
$$

The focus of the descriptive near set theory is to assess similarity in terms of the descriptions of objects within the sets. For each visual element $x_{0}$ in the union of all sub-images $\left(x_{0} \in X \cup Y\right)$, where $X$ and $Y$ are two objects (images), find the tolerance classes with respect to the tolerance relation $\cong_{\phi, \epsilon}$. Tolerance classes are composed of the query points of successive neighborhoods, and then all the tolerance classes containing $x \in X$ are subsets of the neighborhood of $x[14$. Finding tolerance classes is based on the Maximal Clique Enumeration (MCE) approach. This concept consists of using a tree structure to find all the maximal cliques through a depth-first search, where each call to Clique Enumerate creates a new child node. The general idea is to find maximal cliques through a Depth-First Search where the branches are formed based on candidate cliques and the backtracking occurs once a maximal clique has been discovered. This algorithm finds all the tolerance classes. The main idea behind using tolerance classes is the conjecture that when we look at two images, we tend to group image elements based on similarity to the element of interest at the point of gaze.

\section{Fuzzy Similarity Measure}

In this section, we proposed three new fuzzy similarity measures between IT-2 FSs. The notion of a similarity measure can be formalized through quantifying the differences between two objects.

\section{1) Interval Type-2 Fuzzy Nearness Measures 1:}

In a tolerance space view to image correspondence, nearness between sets of describable objects $X, Y$ is defined by comparing the tolerance classes of almost similar objects in a tolerance space that covers both images. The idea behind the nearness measure of Henry and Peters has sought the level of similarity between two variables by omitting the existing correlation between the set of variables, called the tolerance classes. The similarity measures can be grouped into equivalence classes of measures. The Tolerance Fuzzy Nearness Measure between two fuzzy sets $X ; Y$ is based on the concept that equivalent classes formed from objects in the union $Z=X \cup Y$ should be uniformly divided between $X$ and $Y$ if these sets are similar. A Tolerance Fuzzy Nearness Measure (TFNM) is proposed in this paper. By analogy to Type-2 Fuzzy Logic [26], the intersection of any FS will be replaced by minimum. Then based on the Equation 2, TFNM will be defined by the following definition.

Definition: Let $\langle O, F\rangle$ be a perceptual system, with $\epsilon \in \Re_{0}^{+}$and $B \subset F$. Let $X$ and $Y$ be two disjoint sets. A tolerance fuzzy nearness measure (IT2FNM1) between two sets $X$ and $Y$ is determined by:

$$
\operatorname{IT} 2 F N M 1=1-\frac{(\bar{s}+\underline{s})}{2}
$$

where

$$
\begin{aligned}
& \bar{s} \cong_{B, \epsilon}(\tilde{X}, \tilde{Y})=
\end{aligned}
$$

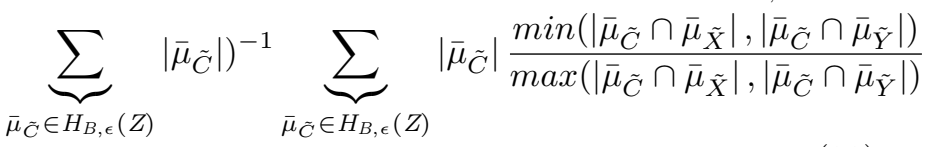


and

$$
\begin{array}{r}
\left.\sum_{\underline{\mu}_{\tilde{C}} \in H_{B, \epsilon}(Z)}\left|\underline{\mu}_{\tilde{C}}\right|\right)^{-1} \cdot \underbrace{\sum_{\underline{\Omega}_{B, \epsilon}}(\tilde{X}, \tilde{Y})=}_{\underline{\mu}_{\tilde{C}} \in H_{B, \epsilon}(Z)} \\
\frac{\min \left(\left|\underline{\mu}_{\tilde{C}} \cap \underline{\mu}_{\tilde{X}}\right|,\left|\underline{\mu}_{\tilde{C}} \cap \underline{\mu}_{\tilde{Y}}\right|\right)}{\max \left(\left|\underline{\mu}_{\tilde{C}} \cap \underline{\mu}_{\tilde{X}}\right|,\left|\underline{\mu}_{\tilde{C}} \cap \underline{\mu}_{\tilde{Y}}\right|\right)}
\end{array}
$$

where $H_{\cong_{B, \epsilon}}(Z)$ is the set of fuzzy tolerance classes. Note that $X, Y$ are pairs of images and $X, Y$ represent sets of describable objects (visual elements) corresponding to images $X, Y$. When the cardinality of a fuzzy set is defined as the sum of the membership values of all the elements in a set (as defined in [44]). The Interval Type-2 Fuzzy Nearness Measure is the mean of Tolerance Type-2 Fuzzyupper Nearness Measure and Interval Type-2 Fuzzy-lower Nearness Measure.

Proof and justification: See Appendix Proof of IT2FNM1 properties.

2) Interval Type-2 Fuzzy Nearness Measures 2:

As noted in Section 2, for 2 IT-2 FSs $A$ and $B$, Jaccard's similarity is represented by the intersection between A and $\mathrm{B}$ divided by the maximum of their union. According to Henry's metric-free description-based nearness measure [16], so it is a measure of similarity since

$$
\text { Similarity }=1-\text { Distance }
$$

according to 37 . Therefore, this measure is equivalent to that of the Jaccard similarity measure. By analogy to fuzzy Logic [26] and according to set operations, the absolute difference of $(X$ and $Y$ ) and the algebraic sum of $X$ and $Y$ reduce to the union and the intersection between $A$ and $B$ respectively. Thus, based on the equation of the nearness measure 2 and as the false membership function will be replaced by $1 \mathrm{t}(x)$, the equation 12 will be transformed as:

$$
I T 2 F N M 2=1-\frac{(\bar{s}+\underline{s})}{2}
$$

where

$$
\begin{aligned}
& (2 \times \underbrace{}_{\bar{\mu}_{C} \in H_{B, \epsilon}(Z)}\left|\bar{\mu}_{C}\right|)^{-1} \cdot \underbrace{\sum_{\cong_{B, \epsilon}}(X, Y)=}_{\bar{\mu}_{C} \in H_{B, \epsilon}(Z)}\left|\bar{\mu}_{C}\right| \\
& \left(\frac{\left|\min \left(\left|\bar{\mu}_{C} \cap \bar{\mu}_{X}\right|,\left|\bar{\mu}_{C} \cap \bar{\mu}_{Y}\right|\right)\right|}{\max \left(\left|\bar{\mu}_{C} \cap \bar{\mu}_{X}\right|,\left|\bar{\mu}_{C} \cap \bar{\mu}_{Y}\right|\right)}+\right. \\
& \left.\frac{\left|\min \left(\left|\bar{\mu}_{C} \cap \bar{\mu}_{X}\right|,\left|\bar{\mu}_{C} \cap \bar{\mu}_{Y}\right|\right)\right|}{2-\max \left(\left|\bar{\mu}_{C} \cap \bar{\mu}_{X}\right|,\left|\bar{\mu}_{C} \cap \bar{\mu}_{Y}\right|\right)}\right)
\end{aligned}
$$

and

$$
\begin{aligned}
& \underline{s} \cong_{B, \epsilon}(X, Y)= \\
& \begin{aligned}
(2 \times \underbrace{}_{\underline{\mu}_{C} \in H_{B, \epsilon}(Z)}\left|\underline{\mu}_{C}\right|)^{-1} \cdot \underbrace{\sum_{B, \epsilon}}_{\underline{\mu}_{C} \in H_{B, \epsilon}(Z)}\left|\underline{\mu}_{C}\right| \\
\\
\left(\frac{\left|\min \left(\left|\underline{\mu}_{C} \cap \underline{\mu}_{X}\right|,\left|\underline{\mu}_{C} \cap \underline{\mu}_{Y}\right|\right)\right|}{\max \left(\left|\underline{\mu}_{C} \cap \underline{\mu}_{X}\right|,\left|\underline{\mu}_{C} \cap \underline{\mu}_{Y}\right|\right)}+\right. \\
\left.\frac{\left|\min \left(\left|\underline{\mu}_{C} \cap \underline{\mu}_{X}\right|,\left|\underline{\mu}_{C} \cap \underline{\mu}_{Y}\right|\right)\right|}{2-\max \left(\left|\underline{\mu}_{C} \cap \underline{\mu}_{X}\right|,\left|\underline{\mu}_{C} \cap \underline{\mu}_{Y}\right|\right)}\right)
\end{aligned}
\end{aligned}
$$

where $\bar{s}$ and $\underline{s}$ defined the upper and lower values respectively.

Proof and justification: See Appendix Proof of IT2FNM2 properties.

3) Interval Type-2 Fuzzy Nearness Measures 3: By the same principle, the Interval Type-2 Fuzzy similarity Measure $\mathrm{S} 3$ is defined by the generalization from the similarity measure presented in Equation 15 between IFS. The latter are computing the mean of lower and upper values. For two IT-2 FSs X and Y, the following formula is hence obtained:

$$
I T 2 F N M 3=1-\frac{(\bar{s}+\underline{s})}{2}
$$

where

$$
\begin{aligned}
&(2 \times \underbrace{}_{\bar{\mu}_{C} \in H_{B, \epsilon}(Z)}\left|\bar{\mu}_{C}\right|)^{-1} \cdot \underbrace{\sum_{\bigwedge_{B, \epsilon}}(X, Y)=}_{\bar{\mu}_{C} \in H_{B, \epsilon}(Z)}\left|\bar{\mu}_{C}\right| \\
&\left(\frac{\left|\min \left(\left|\bar{\mu}_{C} \cap \bar{\mu}_{X}\right|,\left|\bar{\mu}_{C} \cap \bar{\mu}_{Y}\right|\right)\right|}{\max \left(\left|\bar{\mu}_{C} \cap \bar{\mu}_{X}\right|,\left|\bar{\mu}_{C} \cap \bar{\mu}_{Y}\right|\right)}+\right. \\
&\left.\frac{\left|\min \left(\left|\bar{\mu}_{C} \cap \bar{\mu}_{Y}\right|,\left|\bar{\mu}_{C} \cap \bar{\mu}_{X}\right|\right)\right|}{\max \left(\left|\bar{\mu}_{C} \cap \bar{\mu}_{Y}\right|,\left|\bar{\mu}_{C} \cap \bar{\mu}_{X}\right|\right)}\right)
\end{aligned}
$$

and

$$
\begin{array}{r}
(2 \times \underbrace{}_{\underline{\mu}_{C} \in H_{B, \epsilon}(Z)}\left|\underline{\mu}_{C}\right|)^{-1} \cdot \underbrace{\sum_{\underline{s}} \underline{\underline{B}}_{B, \epsilon}(X, Y)=}_{\underline{\mu}_{C} \in H_{B, \epsilon}(Z)}\left|\underline{\mu}_{C}\right| \\
\left(\frac{\left|\min \left(\left|\underline{\mu}_{C} \cap \underline{\mu}_{X}\right|,\left|\underline{\mu}_{C} \cap \underline{\mu}_{Y}\right|\right)\right|}{\max \left(\left|\underline{\mu}_{C} \cap \underline{\mu}_{X}\right|,\left|\underline{\mu}_{C} \cap \underline{\mu}_{Y}\right|\right)}+\right. \\
\left.\frac{\left|\min \left(\left|\underline{\mu}_{C} \cap \underline{\mu}_{Y}\right|,\left|\underline{\mu}_{C} \cap \underline{\mu}_{X}\right|\right)\right|}{\max \left(\left|\underline{\mu}_{C} \cap \underline{\mu}_{Y}\right|,\left|\underline{\mu}_{C} \cap \underline{\mu}_{X}\right|\right)}\right)
\end{array}
$$

where $\bar{s}$ and $\underline{s}$ defined the upper and lower values respectively.

Proof and justification: See Appendix Proof of IT2FNM3 properties.

The best similarity measure provides the largest number of relevant images. The measure of similarity between images is assimilated to a calculation of the distance between the descriptor vector of the query image and that of an image of the base. Both the distance is small as the two images are similar.

\section{Returning query results}

The system returns the result of the search as a list of ordered images according to the similarity between their descriptors and the descriptor of the query image. The effectiveness of the search is evaluated according to the number of images relevant and irrelevant to the query, found in a database: a search making it possible to find, in an image database, all the images relevant to the request, and no irrelevant image, is perfectly effective. 


\section{EXPERIMENTS AND RESULTS}

In this section, we describe the image data sets used. Then, we deal with the experimental results of the proposed method. Finally, we discussed the comparison of these results with the results of other CBIR systems.

\section{A. DataBase}

The performance is evaluated on the Corel-1K, SIMPLIcity, Caltech-101 and ImageNet image repository. The Corel-1K image repository contains 1000 images in the form of 10 semantic categories and each semantic category contains 100 images with resolution sizes of $128 \times 192$ or $192 \times 128$. The SIMPLIcity database contains 1000 natural color images available for download from 46. This database is numbered between 0 to 999 and divided into 10 conceptually different categories (named here as target sets $\mathrm{C} 0$ to $\mathrm{C} 9$ ). Images are $384 \times 256$ pixels. The original CALTECH database consists of images of 101 categories. Each category contains 40 to 800 image samples. Each image is roughly $300 \times 200$ in size. The Image-Net 47 is a large-scale image database. It is used for indexing and retrieval complex and multi-category images. It contains more than $100 \mathrm{k}$ datasets (i.e. called synsets). In this study, we used only the 20 most popular synsets including 10000 images: airplane, minivan, train, car, fire engine, tank, army, musket, tiger, quail, tortoise, snake, ladle, box, pot, towel, dam, mountain, fisherman and volcano. Fig. 2 displays sample images from Corel-1k, SIMPLIcity, Caltech-101 and ImageNet databases. Any image from the database can be selected as a query image and compared to all images in the database using either the fuzzy nearness approaches mentioned above. The experimentation con-

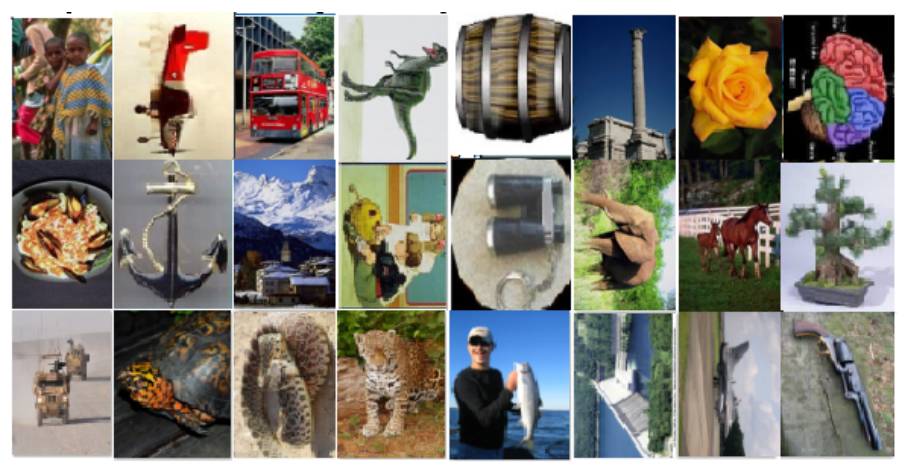

Fig. 2. Sample images from Corel 10k, SIMPLIcity, Caltech-101 and ImageNet databases

sists of calculating the similarity measures between each query image and all images. Subsequently, the images will be sorted based on their similarity to the query image. The experimentation is performed using each one of the proposed similarity measures in previous chapters. The experimentation was generated using $\epsilon=0,3$ as a value produced the best results in [14].

Three different methods were used in this experiment to evaluate precision and recall of the image retrieval. In the first evaluation method, mean average precision for each category between the proposed measures on the basis of the formula 19, 23 and 26. In a second method of retrieval evaluation, both precision and recall were calculated at each number of the 100 most similar images (each category) and the values of precision were plotted against recall. In a third evaluation method Comparison of average precision for each category between the proposed measures, results published in [5]-9] on the SIMPLIcity, corel-1k, caltech-101 and ImageNet databases. The experimental results were provided by different approaches :

- BFNS : Our proposal (Beta Fuzzy Near System) is based on the beta fuzzy Near sets approach with fuzzy nearness measure 14].

- IT2BFNS1 : Our proposal (Interval Type-2 Beta Fuzzy Near System 1) is based on the interval type2 beta fuzzy Near set approach with fuzzy nearness measure 1.

- IT2BFNS2 : Our proposal (Interval Type-2 Beta Fuzzy Near System 2) is based on the interval type2 beta fuzzy Near set approach with fuzzy nearness measure2.

- IT2BFNS3 : Our proposal (Interval Type-2 Beta Fuzzy Near System 3) is based on the interval type2 beta fuzzy Near set approach with fuzzy nearness measure 3 .

We present some examples of experimental results obtained from the measures studied.

\section{B. Performance Measurement for Similar Image Retrieval}

To assess the effectiveness of our system, we interested in calculating the two most commonly used measures. For a given query image, a retrieved image is considered relevant if and only if it belongs to the same image category as the query image. The precision is the number of relevant images retrieved in relation to the total number of images proposed by the search engine for a given query. Precision is calculated using the following formula:

$$
\text { precision }=\frac{\mid\{\text { relevant images }\} \cap\{\text { retrieved images }\} \mid}{\{\text { retrieved images }\}}
$$

The recall is the ratio between the number of relevant images in the set of images found and the number of relevant images in the image base. Recall is defined as follows:

$$
\text { Recall }=\frac{\mid\{\text { relevant images }\} \cap\{\text { retrieved images }\} \mid}{\{\text { relevant images }\}}
$$

In practice, in order to evaluate the system, we use several queries. It is therefore necessary to calculate the AP (Average Precision) for all the requests corresponding to each level of recall according to the following formula:

$$
A P=\frac{\sum_{i}(\text { precision of the query } i)}{\text { Number of requests }}
$$

This measurement is calculated on all queries and represents the arithmetic mean of the different precision of each 
category. To evaluate the experiments, the AP (Average Precision) has been calculated, the different results will be presented. The Mean Average Precision (MAP) is the mean of all the APs of the different categories. Precision and Recall are interesting for a final evaluation of the best image of one category, however for larger evaluation purposes, we consider the Precision/Recall curve. This curve is the set of all the couples (Precision, Recall) for each number of images returned by the system. The curve always starts from the top left $(1,0)$ and ends in the bottom right $(0,1)$. Between these two points, the curve decreases regularly. A good Precision/Recall curve is a curve that decreases slowly, which means that at the same time, the system returns a lot of relevant images and few of them are lost.

\section{Results and Analysis}

In the first experimentation, the retrieval results of the average precision and average recall are reported in Table [1 As seen in this table, IT2BFNS3 outperforms IT2BFNS1 and IT2BFNS2 similarity measures in all categories, and we show that, for the proposed IT2BFNS3, three image classes 'Elephant', 'Flowers' and 'Horses' have attained $100 \%$ average precision.

The second experimentation have been performed on Caltech-101 data collection. We selected randomly fifteen categories; the contents include airplanes, ferry, camera, brain, minaret, motorbrikes, etc. To use the Caltech database in a similar manner as for the SIMPLIcity database, 100 images of each class are randomly selected. The average precision and recall rates for 15 categories of the Caltech-101 database are shown in Table II Similarly, IT2BFNS3 provides better precision in those categories. The recall rates for Caltech-101 are also promising. Most of the categories have high recall rates, while a few have average rates. The Windsor chair and camera have average rates. The mean average precision obtained for this benchmark is $88.7 \%$, and the mean average recall is $50.05 \%$.

The third experimentation is done for evaluating the proposed fuzzy similarity measures on the Corel-1k database. The average precisions and recalls of all categories in this database are reported in Table III

Results show that IT2BFNS3 has a higher average precision rate in some categories, and a significantly better average recall rate. The recall rate is improved by $19.14 \%$ over those from the existing measures. IT2BFNS3 outperforms other measures by at least $5.51 \%$ in terms of average precision. The horses, flower and elephant categories are relatively easy to retrieved, and most of the existing methods provide better results in these categories, it reaches a rate of $100 \%$. Table IV shows a comparison between performance analysis of proposed and existing measures in terms of average precision and average recall on 20 catégories of Image-Net datasets. Therefore, we can draw the following conclusions; first, the experiments performed on Image-Net are a very challenging task. In fact, the average precision rates obtained for each category are around $63 \%$ which implies that they achieve an equivalent performance for all proposed measures. On the other hand, the proposed IT2FBNS3 method achieves the highest precision rates in some categories since Image-Net synsets are difficult to categorize. Secondly, The average recall rates obtained for each category are around $37 \%$, where IT2FBNS1 achieves the best performance with $39.49 \%$ performance. Then, the image-Net shows average results due to its complex nature of images which falls into the different semantic groups at the same time.

To further the understanding, the second experimentation is done by presenting in fig. 3(a); an example query from the beach category of SIMPLIcity database. Fig. 3(b) shows the first 20 images extracted from the best search of the query are sorted and displayed according to the nearness measure. In this figure, IT2FBNS3 (first row), IT2FBNS2 (second row), IT2FBNS1 (thrid row), and FBNS (four row), are shown. The first row contains only 13 images from the class and 7 irrelevant images of other classes. Then IT2BFNS3 method leaves less mistakes then other measures. Fig. 3(c) shown the precision and recall curve for the query with different images retrieved for this database. Some of the curves have an acute inflection point. These points represent the location where the remaining IT2BFNM values for a particular request become null. It can be seen that images in category "beach" can be retrieved with very high precision with the measure IT2BFNM3. Fig. 4 is another example of an image of Imag-Net database. Fig. 4(b) shows that IT2FBNS3 give more relevant images then other methods. Fig. 4 (c) shown the precision and recall curve. It can be seen also that IT2BFNM3 method have a better precision. To demonstrate the effectiveness of the new similarity measure, the nearness measure had compared with three different kinds of similarity measures in [15]; Tolerance Nearness Measure (TNM), Earth Mover's Distance (EMD), and Integrated Region Matching (IRM). Hence, it is experimentally verified that TNM is an effective similarity measure in image retrieval. Table $\mathrm{V}$ encompasses the results obtained for different distance metrics and with recent CBIR algorithms [8], [48] using the SIMPLIcity, Corel-10K, and Caltech-101 databases. This, clearly shows that, the Euclidean distance metric delivers the better result than other metrics. It produced $69 \%$ precision and $7.5 \%$ recall in SIMPLIcity, $36.83 \%$ precision and $7.5 \%$ recall in Corel-10k, and $65 \%$ precision and $7.5 \%$ recall in Caltech-101. In the third experimentation, the best results of our proposed similarity measure are evaluated and compared to them in [5]-9]. The numerical results of the average precision are reported in table VI. As seen in this table, the average precision of our proposed measures on Corel- $1 \mathrm{k}$ is $88.65 \%$. This is significantly better than others. Table IV-C shows the average precision rate for the best proposed metric and existing research metrics for SIMPLIcity database. The existing methods also report good results in many categories but the proposed method reports the highest mean average precision. 


\begin{tabular}{|l|l|l|l|l|l|l|l|l|}
\hline Categories & BFNS & IT2BFNS1 & IT2BFNS2 & IT2BFNS3 \\
\hline & Precision(\%) & Recall(\%) & Precision(\%) & Recall(\%) & Precision(\%) & Recall(\%) & Precision(\%) & Recall(\%) \\
\hline Africa & 76.3 & 53 & 16.51 & 50.49 & 21,84 & 50.49 & 79.02 & 55 \\
\hline Beach & 72.5 & 65 & 28.60 & 78 & 22.27 & 83 & 7693 & 91 \\
\hline Building & 76.2 & 61 & 24.33 & 68 & 20.59 & 75 & 75.74 & 87 \\
\hline Bus & 92.3 & 68 & 25.10 & 74 & 23.47 & 85 & 91,91 & 92 \\
\hline Dinosaur & 100 & 91 & 54.82 & 91 & 55.43 & 97 & 89.72 & 100 \\
\hline Elephant & 74.8 & 61 & 69.05 & 69 & 100 & 75 & 100 & 83 \\
\hline Flower & 89.2 & 55 & 100 & 65 & 100 & 71 & 100 & 81 \\
\hline Horse & 100 & 58 & 100 & 65 & 98 & 76 & 100 & 80 \\
\hline Mountain & 66.8 & 53 & 58.85 & 61 & 80 & 69 & 90 & 76 \\
\hline Food & 68.7 & 64 & 80.35 & 71 & 87.05 & 75 & 92.02 & 92 \\
\hline Average & 81.18 & 60 & 82.35 & 67 & 84.76 & 66 & 88.2 & 79 \\
\hline
\end{tabular}

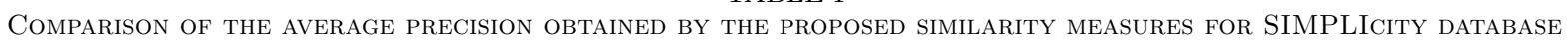

\begin{tabular}{|c|c|c|c|c|c|c|c|c|}
\hline \multirow[t]{2}{*}{ Categories } & \multicolumn{2}{|l|}{ BFNS } & \multicolumn{2}{|l|}{ IT2BFNS1 } & \multicolumn{2}{|l|}{ IT2BFNS2 } & \multicolumn{2}{|l|}{ IT2BFNS3 } \\
\hline & Precision(\%) & Recall $(\%)$ & Precision(\%) & Recall $(\%)$ & Precision(\%) & Recall(\%) & Precision(\%) & Recall $(\%)$ \\
\hline Airplanes & 76 & 48.02 & 95 & 51.1 & 72 & 54 & 94 & 57.18 \\
\hline Ferry & 75 & 32.05 & 66 & 34 & 66 & 37.11 & 88 & 41 \\
\hline Camera & 77 & 48.2 & 85 & 51 & 72 & 54.02 & 84 & 57.11 \\
\hline Brain & 71 & 44.8 & 79 & 51.2 & 70 & 54.5 & 91 & 56.22 \\
\hline Cougarface & 72 & 45 & 83 & 49.9 & 71 & 51.05 & 87 & 45 \\
\hline Grad piano & 76 & 44.05 & 90 & 44.05 & 73 & 53 & 92 & 56 \\
\hline Dalmation & 70 & 39 & 73 & 43 & 68 & 46 & 90 & 49 \\
\hline Dollar bill & 76 & 37 & 35 & 41.78 & 40 & 44.21 & 86 & 47.11 \\
\hline Starfish & 77 & 32 & 45 & 37 & 74 & 42.2 & 88 & 47.68 \\
\hline Soccer ball & 74 & 36.08 & 82 & 41.22 & 71 & 43.05 & 87 & 51.55 \\
\hline Minaret & 74 & 31.46 & 66 & 33.01 & 79 & 37.5 & 88 & 45.05 \\
\hline Motorbikes & 73 & 35.88 & 78 & 37 & 72 & 41.78 & 90 & 43 \\
\hline Revolver & 70 & 36 & 73 & 39.03 & 71 & 42 & 85 & 46.66 \\
\hline Sunflower & 50 & 35.29 & 66 & 38.44 & 68 & 43 & 92 & 50.05 \\
\hline Windsorchair & 51 & 35.29 & 74 & 38.44 & 73 & 43 & 84 & 50.05 \\
\hline \begin{tabular}{|l|} 
Average \\
\end{tabular} & 71 & 35.29 & 69.5 & 38.44 & 72.8 & 43 & 88.7 & 50.05 \\
\hline
\end{tabular}

Comparison of the aVerage precision obtained by the proposed similarity measures on the Caltech-101 Database

\begin{tabular}{|l|l|l|l|l|l|l|l|l|}
\hline Categories & BFNS & \multicolumn{2}{l|}{ IT2BFNS1 } & IT2BFNS2 & IT2BFNS3 \\
\hline & Precision(\%) & Recall(\%) & Precision(\%) & Recall(\%) & Precision(\%) & Recall(\%) & Precision(\%) & Recall(\%) \\
\hline Africa & 73.3 & 2.05 & 66.5 & 7.49 & 72 & 7.95 & 89.02 & 20.03 \\
\hline Beach & 59.5 & 12.05 & 60 & 4.04 & 65 & 9.40 & 70.5 & 14.05 \\
\hline Building & 67.2 & 2.15 & 66.9 & 4.84 & 72 & 7.22 & 78.14 & 18.20 \\
\hline Bus & 72.3 & 1.72 & 76 & 10.82 & 85 & 16.68 & 92.3 & 20.50 \\
\hline Dinosaur & 99.5 & 19.93 & 96.2 & 18.72 & 98.8 & 19.83 & 98 & 23.47 \\
\hline Elephant & 84.1 & 9.93 & 98 & 9.08 & 100 & 12.84 & 100 & 14.12 \\
\hline Flower & 82.3 & 17.10 & 88 & 13.54 & 84 & 17.26 & 100 & 23.12 \\
\hline Horse & 100 & 18.64 & 100 & 10.19 & 98 & 18.85 & 100 & 25.78 \\
\hline Mountain & 66.8 & 9.83 & 58.85 & 4.52 & 80 & 10.51 & 82 & 14.95 \\
\hline Food & 78.7 & 2.23 & 80.35 & 6.12 & 80 & 10.32 & 80 & 17.25 \\
\hline Average & 78.34 & 9.65 & 79.03 & 8.94 & 83.48 & 13.09 & 88.65 & 19.14 \\
\hline
\end{tabular}

Comparison of the average precision obtained by the proposed Similarity measures for Corel-1K Database

\section{CONCLUSIONS AND FUTURE WORK}

In this paper, an Interval Type-2 Beta Fuzzy Sets approach to Content-Based Image Retrieval was presented. This work has improved the feature visual used in contentbased image retrieval application by introducing the Interval Type-2 Beta function. The usage of fuzzy-similarity relations is proposed to ameliorate the performance of similarity for image retrieval. Three Interval Type-2 measures were proposed as an extension of some nearness ones. Mathematical Proves were done to show that the measures advanced are really proximity ones. The proposed measures are working on a list of features, unlike classification based CBIR which requires a large training set. Experimental results show that the proposed approach with IT2FBNS3 measure gives a higher performance that achieves a precision rate of almost $90 \%$ over the SIMPLIcity, Corel-1k, and Caltech-101 datasets and a precision rate of almost $65 \%$ over the big Image-Net datasets. The CBIR problem is still a challenging problem. One of the biggest challenges in CBIR is that, in many cases, there is little correlation between the low-level image features and high-level semantics. Indeed, current CBIRs still lack the accuracy of the relevant image due to incorrect selection of feature extraction methods. Consequently, implementing more visual features for comparing images and to incorporate more powerful feature extraction/selection methods is 


\begin{tabular}{|c|c|c|c|c|c|c|c|c|}
\hline Categories & \multicolumn{2}{|l|}{ BFNS } & \multicolumn{2}{|l|}{ IT2BFNS1 } & \multicolumn{2}{|l|}{ IT2BFNS2 } & \multicolumn{2}{|l|}{ IT2BFNS3 } \\
\hline & Precision $(\%)$ & Recall(\%) & Precision $(\%)$ & Recall(\%) & Precision(\%) & Recall(\%) & Precision $(\%)$ & Recall(\%) \\
\hline Airliner & 68 & 34.6 & 72.3 & 43 & 72 & 44.2 & 70.8 & 43.6 \\
\hline minivan & 66.3 & 33.2 & 62.4 & 40 & 51.7 & 32.3 & 67.8 & 32.3 \\
\hline train & 78 & 44.5 & 77 & 44.5 & 76 & 44.5 & 77 & 44.5 \\
\hline car & 72.5 & 34.8 & 71 & 34.5 & 72 & 34.5 & 72 & 34.5 \\
\hline fire-engine & 68 & 44.5 & 73 & 44.5 & 69.8 & 44.5 & 77.3 & 44.5 \\
\hline $\operatorname{tank}$ & 77.4 & 38.2 & 75.6 & 38.2 & 87 & 38.2 & 78.2 & 38.2 \\
\hline army & 72 & 44.2 & 68.2 & 38.2 & 70 & 44.2 & 72 & 38.2 \\
\hline musket & 56.6 & 32.5 & 44.8 & 32.5 & 42.6 & 32.5 & 55.5 & 32.5 \\
\hline tiger & 66 & 29.52 & 66.8 & 32.5 & 66 & 29.52 & 62.8 & 44.5 \\
\hline quail & 54.3 & 34.08 & 60.73 & 29.8 & 58.9 & 32.5 & 57.8 & 32.5 \\
\hline tortoise & 52 & 32.5 & 52 & 32.5 & 51.7 & 37.5 & 52.8 & 32.5 \\
\hline snake & 49.2 & 32.5 & 52 & 32.5 & 52 & 32.5 & 50.8 & 32.5 \\
\hline ladle & 72 & 37.5 & 73 & 42.6 & 72 & 42.6 & 73 & 42.6 \\
\hline box & 66.7 & 37.5 & 66 & 42.6 & 56.8 & 43 & 68 & 37.5 \\
\hline pot & 44.7 & 42.6 & 34.5 & 37.5 & 45.6 & 32.5 & 42.2 & 32.5 \\
\hline towel & 70 & 37.5 & 68.2 & 32.5 & 72.8 & 43 & 74.5 & 32.5 \\
\hline dam & 66.7 & 42.6 & 70 & 38.4 & 71 & 32.5 & 72.8 & 38.4 \\
\hline mountain & 67 & 32.5 & 70 & 38.4 & 73 & 32.5 & 72 & 38.4 \\
\hline fisherman & 51 & 35.29 & 64 & 38.4 & 66 & 38.4 & 67.7 & 35.29 \\
\hline volcano & 48.2 & 35.29 & 54 & 38.4 & 52.7 & 32.5 & 52 & 32.5 \\
\hline Average & 63.3 & 36.77 & 63.77 & 39.49 & 63.98 & 37.19 & 63.95 & 36.99 \\
\hline
\end{tabular}

Comparison of the aVerage precision obtained by the proposed similarity measures on the Image-Net database

\begin{tabular}{|c|c|c|c|c|}
\hline \multirow{2}{*}{ database } & \multirow{2}{*}{ Performance } & \multicolumn{3}{|c|}{ Similarity metrics } \\
\hline & & Manhattan & chi-Square & Euclidean \\
\hline \multirow[t]{2}{*}{ SIMPLIcity } & \multirow{2}{*}{$\begin{array}{l}\text { Precision } \\
\text { Recall }\end{array}$} & 40 & 40 & 69 \\
\hline & & 5 & 5 & 7.5 \\
\hline \multirow[t]{2}{*}{ Corel-10k } & \multirow{2}{*}{$\begin{array}{l}\text { Precision } \\
\text { Recall }\end{array}$} & 43.35 & 46 & 36.83 \\
\hline & & 5.20 & 5.52 & 4.42 \\
\hline \multirow{2}{*}{$\begin{array}{l}\text { Caltech- } \\
101\end{array}$} & \multirow{2}{*}{$\begin{array}{l}\text { Precision } \\
\text { Recall }\end{array}$} & 39 & 29 & 65 \\
\hline & & 5.5 & 4 & 7.5 \\
\hline
\end{tabular}

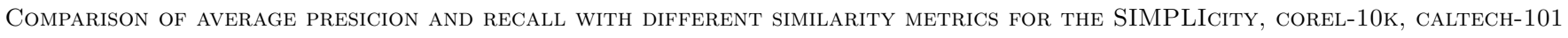
DATABASES

\begin{tabular}{|c|c|c|c|c|c|c|}
\hline set & $\begin{array}{l}\text { Results } \\
\text { reported in } \\
{[7}\end{array}$ & $\begin{array}{l}\text { Results } \\
\text { reported in } \\
8\end{array}$ & $\begin{array}{l}\text { Results } \\
\text { reported in } \\
6\end{array}$ & $\begin{array}{l}\text { Results } \\
\text { reported in } \\
5\end{array}$ & $\begin{array}{l}\text { Results } \\
\text { reported in } \\
{[9}\end{array}$ & $\begin{array}{l}\text { Best result } \\
\text { in our work }\end{array}$ \\
\hline $\mathrm{C} 0$ & $88.05 \%$ & $90 \%$ & $74.80 \%$ & $82 \%$ & $90 \%$ & $89.02 \%$ \\
\hline C1 & $79.5 \%$ & $60 \%$ & $45.85 \%$ & $60 \%$ & $60 \%$ & $70.05 \%$ \\
\hline $\mathrm{C} 2$ & $67.65 \%$ & $90 \%$ & $58.00 \%$ & $67 \%$ & $90 \%$ & $78.14 \%$ \\
\hline C3 & $100 \%$ & $75 \%$ & $77.45 \%$ & $95 \%$ & $100 \%$ & $92.3 \%$ \\
\hline $\mathrm{C} 4$ & $100 \%$ & $100 \%$ & $99.75 \%$ & $100 \%$ & $75 \%$ & $95 \%$ \\
\hline $\mathrm{C} 5$ & $93.10 \%$ & $70 \%$ & $61.60 \%$ & $95 \%$ & $100 \%$ & $100 \%$ \\
\hline C6 & $100 \%$ & $\tilde{9} 0 \%$ & $80.55 \%$ & $100 \%$ & $70 \%$ & $100 \%$ \\
\hline $\mathrm{C} 7$ & $100 \%$ & $100 \%$ & $94.90 \%$ & $100 \%$ & $90 \%$ & $100 \%$ \\
\hline $\mathrm{C} 8$ & $77.75 \%$ & $70 \%$ & $45.05 \%$ & $63 \%$ & $100 \%$ & $82 \%$ \\
\hline C9 & $89.30 \%$ & $90 \%$ & $72.80 \%$ & $71 \%$ & $70 \%$ & $80 \%$ \\
\hline average & $89.50 \%$ & $83.5 \%$ & $71.05 \%$ & $71.05 \%$ & $84.5 \%$ & $88.65 \%$ \\
\hline
\end{tabular}

Comparison of average presicion of Each Category between some techniques on the Corel-1K database

challenging. Also, the development a content-based image retrieval system architecture to support querying very large image databases for a wide variety of data sets in different domain will be good solution. Investigation of these problems is part of our future work.

\section{ACKNOWLEDGMENT}

The research leading to these results has received funding from the Ministry of Higher Education and Scientific Research of Tunisia under the grant agreement number LR11ES48.

\section{REFERENCES}

[1] S. Chavda and M. Goyani, "Content-based image retrieval: The state of the art," International Journal of Next-Generation Computing, vol. 10, no. 3, 2019.

[2] M. Flickner, H. Sawhney, W. Niblack, J. Ashley, Q. Huang, B. Dom, M. Gorkani, J. Hafner, D. Lee, D. Petkovic et al., "Query by image and video content: The qbic system," computer, vol. 28, no. 9, pp. 23-32, 1995.

[3] J. R. Smith and S.-F. Chang, "Visualseek: a fully automated content-based image query system," in Proceedings of the fourth ACM international conference on Multimedia, 1997, pp. 87-98.

[4] J. Z. Wang, J. Li, and G. Wiederhold, "Simplicity: Semanticssensitive integrated matching for picture libraries," IEEE 


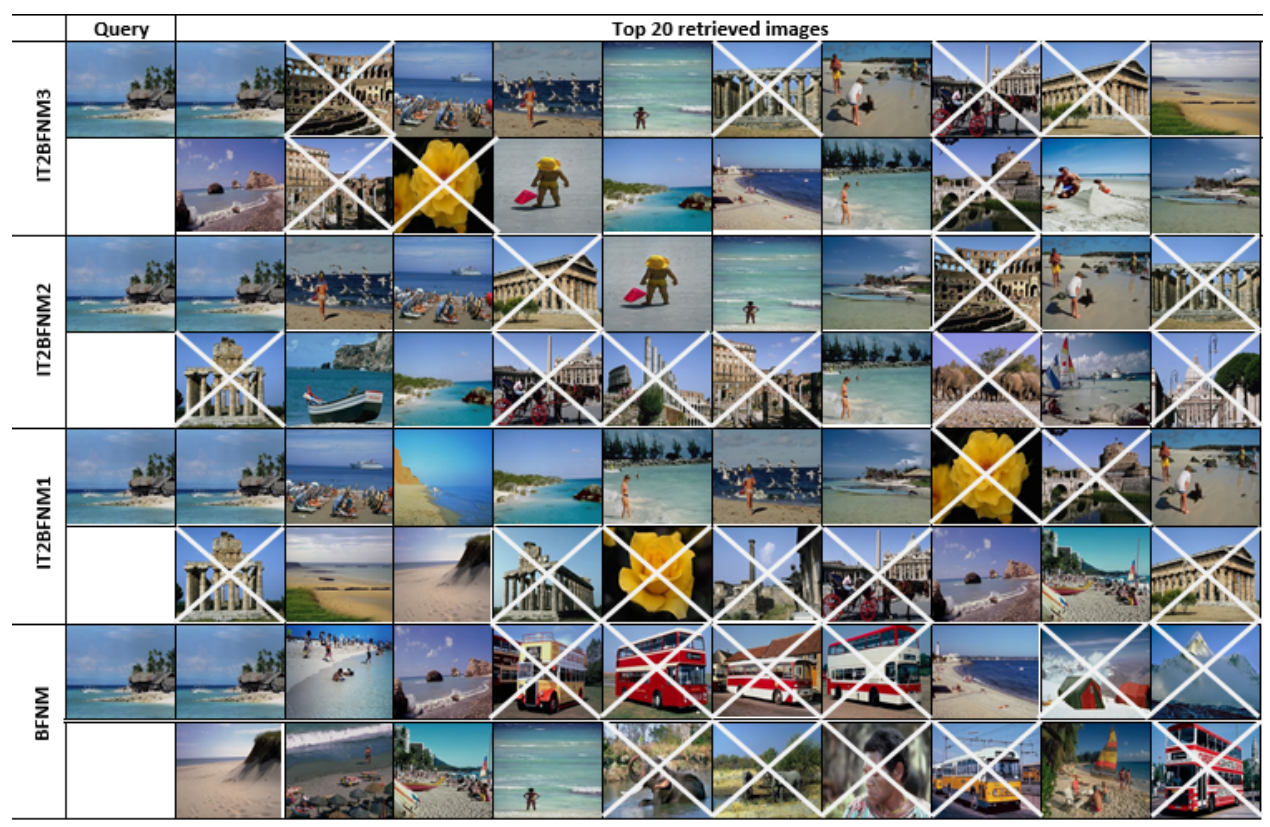

(c) first 20 images retrieved

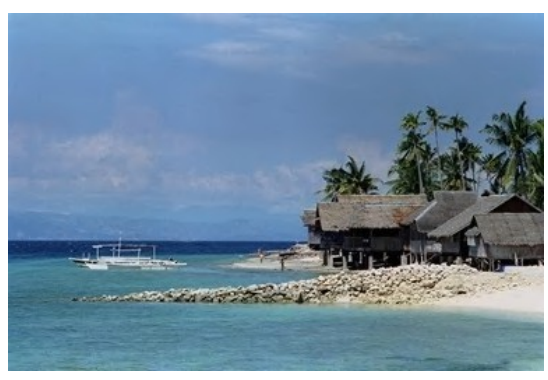

(a) query image

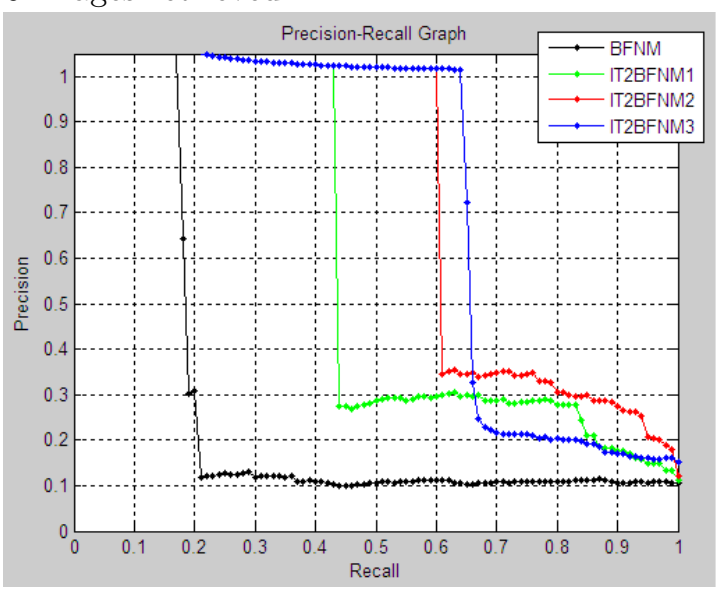

(b) Precision/Recall curve

Fig. 3. Experiment results of the best image : SIMPLIcity database

Transactions on Pattern Analysis and Machine Intelligence, vol. 23, no. 9, pp. 947-963, 2001.

[5] M. I. T. Bella and A. Vasuki, "An efficient image retrieval framework using fused information feature," Computers \& Electrical Engineering, vol. 75, pp. 46-60, 2019.

[6] S. Fadaei, R. Amirfattahi, and M. R. Ahmadzadeh, "New content-based image retrieval system based on optimised integration of dcd, wavelet and curvelet features," IET Image Processing, vol. 11, no. 2, pp. 89-98, 2016.

[7] M. Hamroun, S. Lajmi, H. Nicolas, and I. Amous, "A new method of combining colour, texture and shape features using the genetic algorithm for image retrieval," International Journal of Multimedia Intelligence and Security, vol. 3, no. 3, pp. 293319, 2019.

[8] A. Raza, H. Dawood, H. Dawood, S. Shabbir, R. Mehboob, and A. Banjar, "Correlated primary visual texton histogram features for content base image retrieval," IEEE Access, vol. 6, pp. 46 595-46616, 2018.

[9] K. T. Ahmed, S. Ummesafi, and A. Iqbal, "Content based image retrieval using image features information fusion," Information Fusion, vol. 51, pp. 76-99, 2019.

[10] J. F. Peters and P. Wasilewski, "Foundations of near sets," Information Sciences, vol. 179, no. 18, pp. 3091-3109, 2009.

[11] J. F. Peters and S. Ramanna, "Feature selection: Near set approach," in International Workshop on Mining Complex Data.
Springer, 2007, pp. 57-71.

[12] J. F. Peters, "Tolerance near sets and image correspondence," International Journal of Bio-Inspired Computation, vol. 1, no. 4, pp. 239-245, 2009 .

[13] Y. Ghozzi, N. Baklouti, and A. M. Alimi, "A novel method for resemblance images using near fuzzy set," in 2016 IEEE/ACS 13 th International Conference of Computer Systems and Applications (AICCSA). IEEE, 2016, pp. 1-8.

[14] , "A beta-fuzzy-near-sets approach to research for visually similar content images," in International Conference on Fuzzy Systems, Naples, Italy. IEEE, July 2017, pp. 1-6.

[15] C. J. Henry and S. Ramanna, "Signature-based perceptual nearness: Application of near sets to image retrieval," Mathematics in Computer Science, vol. 7, no. 1, pp. 71-85, $201 \overline{3 .}$

[16] - "Maximal clique enumeration in finding near neighbourhoods." Trans. Rough Sets, vol. 16, pp. 103-124, 2013.

[17] C. J. Henry and S. A. Awais, "A descriptive tolerance nearness measure for performing graph comparison," Fundamenta Informaticae, vol. 163, no. 4, pp. 305-324, 2018.

[18] J. K. Dash and S. Mukhopadhyay, "Similarity learning for texture image retrieval using multiple classifier system," Multimedia Tools and Applications, vol. 77, no. 1, pp. 459-483, 2018.

[19] X. Tang, L. Jiao, and W. J. Emery, "Sar image content retrieval based on fuzzy similarity and relevance feedback," IEEE Journal 


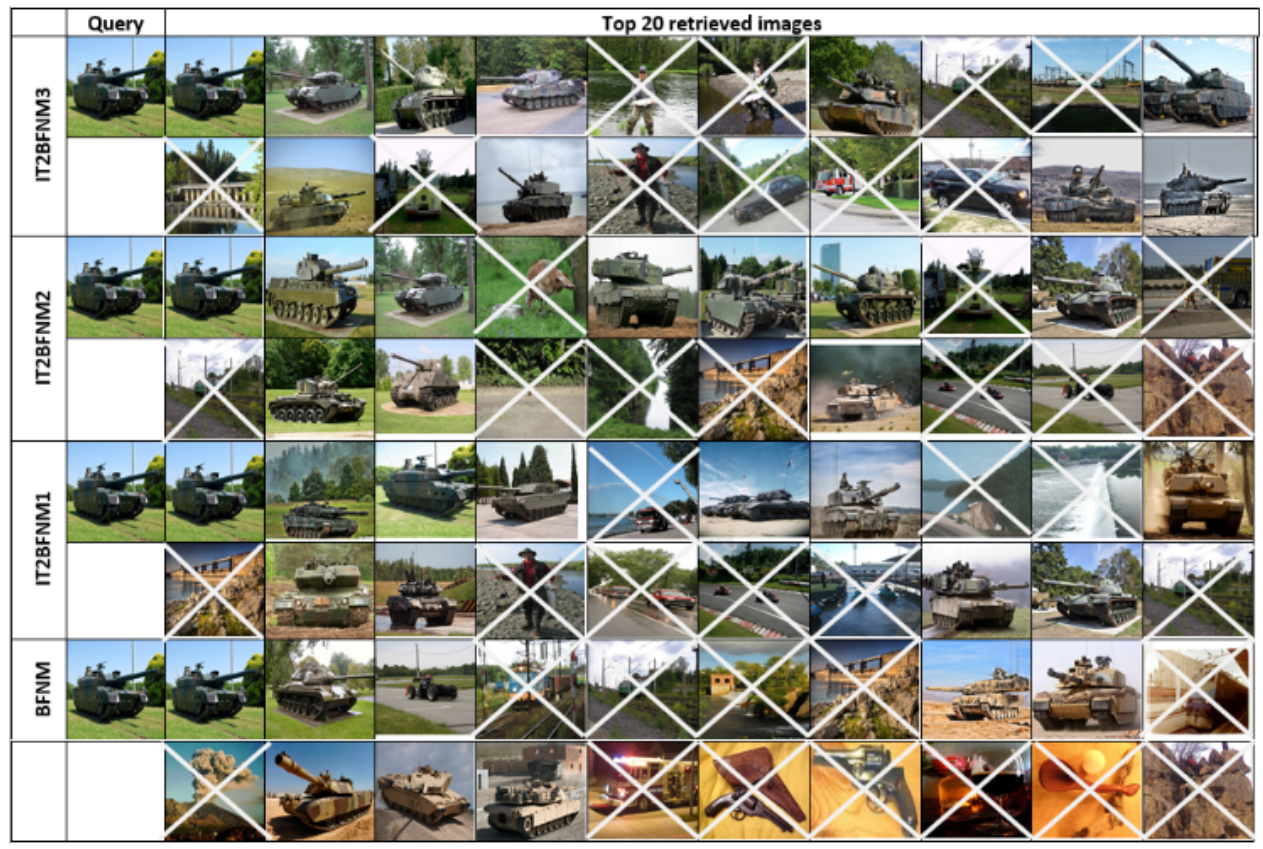

(c) first 20 images retrieved

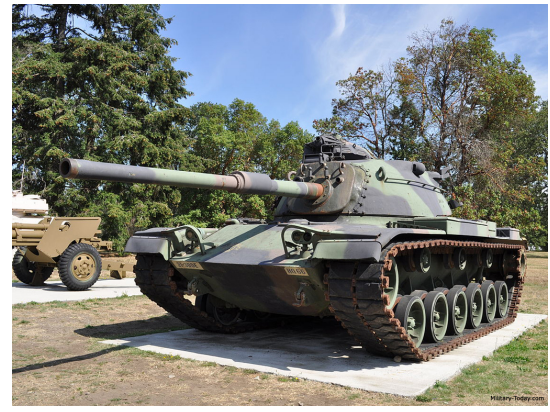

(a) query image

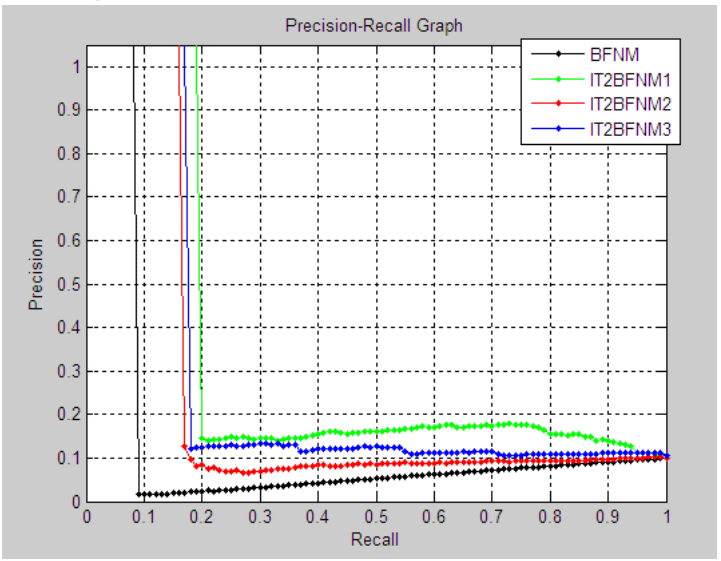

(b) Precision/Recall curve

Fig. 4. Experiment results of an image : Image-Net database

of Selected Topics in Applied Earth Observations and Remote Sensing, vol. 10, no. 5, pp. 1824-1842, 2017.

[20] G. Hesamian, "Fuzzy similarity measure based on fuzzy sets," Control and Cybernetics, vol. 46, 2017.

[21] Y. Song, X. Wang, W. Quan, and W. Huang, "A new approach to construct similarity measure for intuitionistic fuzzy sets," Soft Computing, vol. 23, no. 6, pp. 1985-1998, 2019.

[22] E. Devarasan et al., "Fuzzy techniques for content-based image retrieval," in Feature Dimension Reduction for Content-Based Image Identification. IGI Global, 2018, pp. 41-64.

[23] I. Batyrshin, "Towards a general theory of similarity and association measures: similarity, dissimilarity and correlation functions," Journal of Intelligent \& Fuzzy Systems, vol. 36, no. 4, pp. 2977-3004, 2019.

[24] J. F. Peters, "Fuzzy sets, near sets, and rough sets for your computational intelligence toolbox," in Foundations of Computational Intelligence Volume 2. Springer, 2009, pp. 3-25.

[25] O. Castillo, M. A. Sanchez, C. I. Gonzalez, and G. E. Martinez, "Review of recent type-2 fuzzy image processing applications," Information, vol. 8, no. 3, p. 97, 2017.

[26] L. A. Zadeh, "Fuzzy sets," Information and control, vol. 8, no. 3, pp. 338-353, 1965.

[27] A. Lopez-Maestresalas, L. De Miguel, C. Lopez-Molina, S. Arazuri, H. Bustince, and C. Jarén, "Hyperspectral imaging using notions from type-2 fuzzy sets," Soft Computing, vol. 23, no. 6, pp. 1779-1793, 2019.

[28] L. De Miguel, H. Santos, M. Sesma-Sara, B. Bedregal, A. Jurio, H. Bustince, and H. Hagras, "Type-2 fuzzy entropy sets," IEEE Transactions on Fuzzy Systems, vol. 25, no. 4, pp. 993-1005, 2016.

[29] P. Melin, C. I. Gonzalez, J. R. Castro, O. Mendoza, and O. Castillo, "Edge-detection method for image processing based on generalized type-2 fuzzy logic," IEEE Transactions on Fuzzy Systems, vol. 22 , no. 6 , pp. 1515-1525, 2014.

[30] H. Xing, H. He, D. Hu, T. Jiang, and X. Yu, "An interval type-2 fuzzy sets generation method for remote sensing imagery classification," Computers \& Geosciences, vol. 133, p. 104287, 2019.

[31] V. Singh, N. K. Verma, and Y. Cui, "Type-2 fuzzy pca approach in extracting salient features for molecular cancer diagnostics and prognostics," IEEE transactions on nanobioscience, vol. 18, no. 3, pp. 482-489, 2019.

[32] A. K. Shukla, M. Yadav, S. Kumar, and P. K. Muhuri, "Veracity handling and instance reduction in big data using interval type2 fuzzy sets," Engineering Applications of Artificial Intelligence, vol. 88, p. 103315, 2020.

[33] C. I. Gonzalez, P. Melin, J. R. Castro, and O. Castillo, "Edge detection approach based on type-2 fuzzy images." Journal of 


\begin{tabular}{|l|l|l|l|l|l|}
\hline set & $\begin{array}{l}\text { Results } \\
\text { reported in } \\
\text { 49] }\end{array}$ & $\begin{array}{l}\text { Results } \\
\text { reported in } \\
45]\end{array}$ & $\begin{array}{l}\text { Results } \\
\text { reported in } \\
50]\end{array}$ & $\begin{array}{l}\text { Results } \\
\text { reported in } \\
17\end{array}$ & $\begin{array}{l}\text { Best result } \\
\text { in our work }\end{array}$ \\
\hline C0 & $76.3 \%$ & $83.8 \%$ & $80 \%$ & $66.35 \%$ & $79.02 \%$ \\
\hline C1 & $72.5 \%$ & $73.6 \%$ & $94 \%$ & $52.45 \%$ & $76.93 \%$ \\
\hline C2 & $86.2 \%$ & $85.9 \%$ & $81 \%$ & $43.85 \%$ & $75.74 \%$ \\
\hline C3 & $92.3 \%$ & $71.4 \%$ & $99 \%$ & $69.85 \%$ & $91.91 \%$ \\
\hline C4 & $100 \%$ & $100 \%$ & $100 \%$ & $100 \%$ & $89.72 \%$ \\
\hline C5 & $74.8 \%$ & $69.05 \%$ & $100 \%$ & $94.70 \%$ & $100 \%$ \\
\hline C6 & $89.2 \%$ & $\tilde{100 \%}$ & $100 \%$ & $83.75 \%$ & $100 \%$ \\
\hline C7 & $100 \%$ & $100 \%$ & $98 \%$ & $78.75 \%$ & $100 \%$ \\
\hline C8 & $66.8 \%$ & $58.85 \%$ & $80 \%$ & $64.25 \%$ & $90 \%$ \\
\hline C9 & $78.7 \%$ & $80.35 \%$ & $80 \%$ & $71.3 \%$ & $92.02 \%$ \\
\hline average & $76 \%$ & $73 \%$ & $80.35 \%$ & $72.33 \%$ & $88.2 \%$ \\
\hline
\end{tabular}

COMPARISON OF AVERAGE PRESICION OF EACH CATEGORY BETWEEN SOME TECHNIQUES ON THE SIMPLICITY DATABASE

Multiple-Valued Logic \& Soft Computing, vol. 33, 2019.

[34] C. I. Gonzalez, P. Melin, J. R. Castro, O. Mendoza, and O. Castillo, "An improved sobel edge detection method based on generalized type-2 fuzzy logic," Soft Computing, vol. 20, no. 2, pp. 773-784, 2016.

[35] K. Mittal, A. Jain, K. S. Vaisla, O. Castillo, and J. Kacprzyk, "A comprehensive review on type 2 fuzzy logic applications: Past, present and future," Engineering Applications of Artificial Intelligence, vol. 95, p. 103916, 2020.

[36] D. Wu and J. M. Mendel, "Similarity measures for closed general type-2 fuzzy sets: overview, comparisons, and a geometric approach," IEEE Transactions on Fuzzy Systems, vol. 27, no. 3, pp. $515-526,2018$.

[37] — , "A comparative study of ranking methods, similarity measures and uncertainty measures for interval type-2 fuzzy sets," Information Sciences, vol. 179, no. 8, pp. 1169-1192, 2009.

[38] S. Cherif, N. Baklouti, V. Snasel, and A. M. Alimi, "New fuzzy similarity measures: From intuitionistic to type-2 fuzzy sets," in 2017 IEEE International Conference on Fuzzy Systems (FUZZ-IEEE). IEEE, 2017, pp. 1-6.

[39] J. F. Peters, "Near sets. special theory about nearness of objects," Fundamenta Informaticae, vol. 75 , no. 1-4, pp. 407-433, 2007.

[40] A. M. Alimi, R. Hassine, and M. Selmi, "Beta fuzzy logic systems: Approximation properties in the mimo case," International Journal of Applied Mathematics and Computer Science, vol. 13, pp. 225-238, 2003.

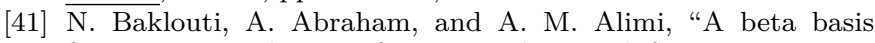
function interval type-2 fuzzy neural network for time series applications," Engineering Applications of Artificial Intelligence, vol. 71, pp. 259-274, 2018.

[42] S. K. Pal and J. F. Peters, Rough fuzzy image analysis: foundations and methodologies. CRC Press, 2010.

[43] J. F. Peters, "Fuzzy sets, near sets, and rough sets for your computational intelligence toolbox." 2009.

[44] L. Baccour and A. M. Alimi, "A comparison of some intuitionistic fuzzy similarity measures applied to handwritten arabic sentences recognition," in Fuzzy Systems, 2009. FUZZ-IEEE 2009. IEEE International Conference on. IEEE, 2009, pp. 13891392 .

[45] A. H. Meghdadi and J. F. Peters, "Perceptual tolerance neighborhood-based similarity in content-based image retrieval and classification," International Journal of Intelligent Computing and Cybernetics, vol. 5, no. 2, pp. 164-185, 2012.

[46] http://cel.archives-ouvertes.fr/cel-00573975/document.

[47] O. Russakovsky, J. Deng, H. Su, J. Krause, S. Satheesh, S. Ma, Z. Huang, A. Karpathy, A. Khosla, M. Bernstein, A. C. Berg, and L. Fei-Fei, "ImageNet Large Scale Visual Recognition Challenge," International Journal of Computer Vision (IJCV), vol. 115, no. 3, pp. 211-252, 2015.

[48] S. P. Rana, M. Dey, and P. Siarry, "Boosting content based image retrieval performance through integration of parametric \& nonparametric approaches," Journal of Visual Communication and Image Representation, vol. 58, pp. 205-219, 2019.

[49] J. Prabhu and J. S. Kumar, "Wavelet based content based image retrieval using color and texture feature extraction by gray level coocurence matrix and color coocurence matrix," Journal of Computer Science, vol. 10, no. 1, p. 15, 2014.

[50] A. ElAdel, R. Ejbali, M. Zaied, and C. B. Amar, "A hybrid approach for content-based image retrieval based on fast beta wavelet network and fuzzy decision support system," Machine Vision and Applications, vol. 27, no. 6, pp. 781-799, Aug 2016. [Online]. Available: https://doi.org/10.1007/s00138-016-0789-z

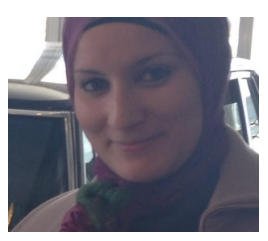

Yosr Ghozzi is a $\mathrm{PhD}$ student in Computer Systems Engineering at the National Engineering School of Sfax (ENIS), since October 2017. She received the M.S. degree in 2014. She is currently a member of the REsearch Group in Intelligent Machines (REGIM). Her research interests include Near sets, CBIR, Neural Fuzzy system, Interval type-2 Fuzzy Logic System.

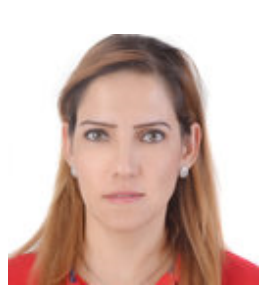

Nesrine Baklouti graduated in Electrical Engineering in 2004. She obtained her Ph.D. degree in Electrical and Computer Engineering in 2013. She is a member of REGIM-Lab on Intelligent Machines. Her research interests include artificial intelligence, analysis and intelligent control and robotic systems. 


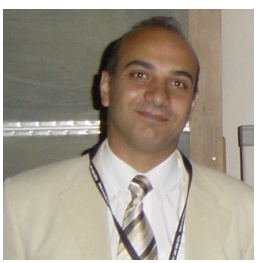

Hani Hagras (M03SM05, F13) He is a Professor in the School of Computer Science and Electronic Engineering, Director of the Computational Intelligence Centre and the Head of the Fuzzy Systems Research Group in the University of Essex, UK. His major research interests are in computational intelligence, notably type-2 fuzzy systems, fuzzy logic, neural networks, genetic algorithms, and evolutionary computation. He is a Fellow of the Institute of Electrical and Electronics Engineers (IEEE) and he is also a Fellow of the Institution of Engineering and Technology (IET (IEE). His research has won numerous prestigious international awards where most recently he was awarded by the IEEE Computational Intelligence Society (CIS), the 2013 Outstanding Paper Award in the IEEE Transactions on Fuzzy Systems and he was also awarded the 2006 Outstanding Paper Award in the IEEE Transactions on Fuzzy Systems.

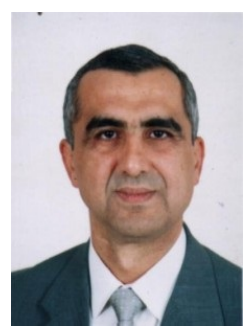

Mounir Ben Ayed (M'01-SM'13) He is an Associate Professor with the Faculty of Science, Computer Science Department, University of Sfax. He teaches mainly data base management system, data warehouse, data mining, software engineering, and human-computer interaction. His current research interests include decision support system based on knowledge discovery from data process. Most of his research are designed and evaluated in the medical field. Dr. Ben Ayed was the Chair, Organization Committee Member, and Technical Committee Member of many national and international conferences. Among the numerous conferences, he organized the Engineering Sciences for Biology and Medicine, ESBM'2013 and ESBM'2015. He created the IEEE-EMBS Tunisia Chapter in 2009. He was the Chair of this chapter from 2009 to 2012, the Vice Chair from 2013 to 2014, and the Chair for the second time from 2015 to 2016.

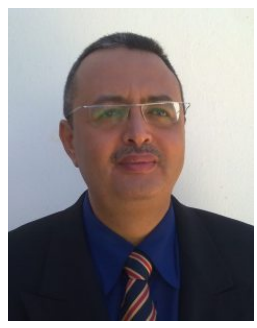

Adel M. Alimi (S'91-M'96-SM'00) He is a Professor of electrical and computer engineering with the University of Sfax. His current research interests include applications of intelligent methods (neural networks, fuzzy logic, and evolutionary algorithms) to pattern recognition, robotic systems, vision systems, industrial processes, intelligent pattern recognition, learning, analysis, and intelligent control of large-scale complex systems. Dr. Alimi is a member of IAPR, INNS, and PRS. He is a member and an Associate Editor of the Editorial Board of many international scientific journals, including Pattern Recognition Letters, Neurocomputing, Neural Processing Letters, International Journal of Image and Graphics, Neural Computing and Applications, International Journal of Robotics and Automation, and International Journal of Systems Science. He was a Guest Editor of several special issues of international journals, including Fuzzy Sets and Systems, Soft Computing, the Journal of Decision Systems, Integrated ComputerAided Engineering, and Systems Analysis Modeling and Simulations. 